\title{
The influence of polarization on the spectral density
}

\author{
Tim J. Zuehlsdorff, ${ }^{1,}$ a) Hanbo Hong, ${ }^{1}$ Liang Shi, ${ }^{1,}$ b) and Christine M. Isborn ${ }^{1, c)}$ \\ Chemistry and Chemical Biology, University of California Merced, Merced, California 95343, \\ USA
}

(Dated: 31 October 2019)

Accurate spectral densities are necessary for computing realistic exciton dynamics and nonlinear optical spectra of chromophores in condensed phase environments, including multi-chromophore pigment-protein systems. However, due to the significant computational cost of computing spectral densities from first principles, requiring many thousands of excited state calculations, most simulations of realistic systems rely on treating the environment as fixed point charges. Here, using a number of representative systems ranging from solvated chromophores to the photoactive yellow protein (PYP), we demonstrate that the quantum mechanical (QM) polarization of the environment is key to obtaining accurate spectral densities and lineshapes within the cumulant framework. We show that the QM environment can enhance or depress the coupling of fast chromophore degrees of freedom to the energy gap, altering the electronic-vibrational coupling and the resulting vibronic progressions in the absorption spectrum. In analyzing the physical origin of peaks in the spectral density, we identify vibrational modes that couple the electron and the hole as being particularly sensitive to the QM screening of the environment. For PYP, we reveal the need for a careful determination of the appropriate QM region to obtain reliable spectral densities. Our results indicate that the QM polarization of the environment can be crucial not just for excitation energies, but also for electronic-vibrational coupling in complex systems, with implications for the correct modeling of linear and nonlinear optical spectroscopy in the condensed phase, as well as energy transfer in pigment-protein complexes.

\section{INTRODUCTION}

The spectral densities that define the system-bath coupling are the foundation of simulations of exciton dynamics of multi-chromophore systems and simulations of nonlinear, ultrafast spectroscopy. ${ }^{1-14}$ Computed spectral densities of complex systems, such as lightharvesting pigment-protein complexes, require many thousands of excited state calculations but reveal fascinating exciton dynamics with the mechanism controlling energy transfer in multi-chromophore systems remaining controversial. ${ }^{15,16}$ The environment surrounding such systems tunes the energy transport; ${ }^{17,18}$ for example, a recent study suggests that the local protein environment plays an essential role in driving incoherent vibronic transport between pigments in a phycobiliprotein complex. $^{12}$

Accurate spectral densities also open the door to improved computation of condensed phase linear absorption spectra through the cumulant framework, which offers multiple advantages over more common techniques. The ensemble approach for computing absorption spectra ${ }^{19-24}$ accounts for specific chromophoreenvironment interactions, but neglects the dynamic coupling that gives rise to vibronic transitions, leading to poor spectral lineshapes. The popular Franck-Condon method $^{25-32}$ expresses the absorption lineshape in terms of nuclear wave function overlaps, often computed by approximating the ground- and excited-state potential en-

\footnotetext{
a) Electronic mail: tzuehlsdorff@ucmerced.edu

b) Electronic mail: lshi4@ucmerced.edu

c) Electronic mail: cisborn@ucmerced.edu
}

ergy surfaces as harmonic around their respective minima. This formalism does not allow for a straightforward inclusion of environmental degrees of freedom in the calculation and the condensed phase environment is often represented in terms of a classical polarizable continuum model ${ }^{33-35}$ that neglects specific chromophoreenvironment interactions. Although recent progress has been made in combining advantages of the ensemble and Franck-Condon approaches, ${ }^{36-38}$ a cumulant expansion of electronic energy gap fluctuations provides an alternative way to express the linear response function. ${ }^{39}$ This cumulant formalism has the advantage that all motion in the complex system can couple to the electronic energy gap and both the environment and the chromophore can be treated on equal footing. This method naturally captures both specific chromophore-environment interactions as well as the dynamic coupling that leads to vibronic fine structure. The cumulant approach has been successfully applied in recent years to study electrontransfer processes, as well as linear spectra of complex systems, with examples including the absorption lineshapes of solvated dyes ${ }^{40}$ and the linear spectrum of a chromophore embedded in the intercalation pocket of DNA. ${ }^{41}$ However, compared to other approaches to computing optical properties of complex systems, such as the classical ensemble and the Franck-Condon approach, the cumulant method is computationally expensive, requiring the calculations of thousands of vertical excitation energies along a sufficiently long molecular dynamics trajectory to converge classical correlation functions of energy gap fluctuations. ${ }^{40,42}$

Given the importance of accurate spectral densities for simulations of linear and nonlinear spectroscopy, as well as exciton dynamics, and the strong dependencies on the 
solvent environment for specific vibrational modes that have been observed experimentally in resonance Raman spectra of push-pull chromophores, ${ }^{43-45}$ there is a clear need to investigate how the treatment of the environment affects the spectral densities. Previous spectral density calculations have predominantly relied on computational savings through modeling the environment as fixed point molecular mechanical (MM) charges. ${ }^{41}$ This MM charge model has proven successful for vibrational spectroscopy and therefore may prove adequate for the electronicvibrational coupling required for spectral densities. This classical condensed phase environment significantly reduces the computational cost of calculations, but may do so at the expense of accurate coupling of the excited state of the chromophore to electronic degrees of freedom of the environment. With the advent of large-scale excited state electronic structure calculations, ${ }^{46-48}$ it has been shown that large portions of the environment should be included in the QM region of the excited state calculation to compute converged excitation energies and ensemble absorption spectra in complex systems. ${ }^{6,19,21,23,24,49-52}$ This spectral shift represents a shift in the average excitation energy, whereas the spectral density measures the fluctuations of the energy gap about this average. It is therefore unclear if the QM polarization of the environment is necessary for simulating accurate spectral densities and the resulting spectral lineshapes.

In the present work, we go beyond the standard model of an MM environment for the computation of spectral densities by using large QM regions for the environment in our excited state calculations. We study systems with varying chromophore-environment interaction strength, including Nile red in different solvents, two anionic chromophores in water, and the photoactive yellow protein. We compute spectral densities of chromophoreenvironment coupling and linear absorption spectra using a range of different QM region sizes when calculating vertical excitation energies. We suggest guidelines to help identify the types of complex systems for which QM polarization of the environment is essential, which we expect to be widely applicable to improved first-principles modeling of linear and nonlinear spectroscopy, as well as exciton dynamics, in complex systems.

\section{THEORY AND COMPUTATIONAL METHODS}

\section{A. Theory}

In the linear-response regime, the absorption spectrum of a system, $\sigma(\omega)$, can be written in terms of the linearresponse function $\chi(t)$ such that ${ }^{39}$ (atomic units used throughout)

$$
\sigma(\omega)=\frac{4 \pi \omega}{3} \int_{-\infty}^{\infty} \mathrm{d} t \chi(t) e^{\mathrm{i} \omega t} .
$$

If the optical response of the system is dominated by a single bright transition, with no contributions from other excited states, the linear-response function can be modeled in terms of an effective two-level electronic system coupled to a bath of nuclear degrees of freedom. Under the Condon approximation, ${ }^{53,54}$ stating that the transition dipole moment $\boldsymbol{\mu}_{e g}$ from the electronic ground- to the excited state is independent of the nuclear degrees of freedom, the linear response function can be written in terms of a cumulant expansion of fluctuations of the energy gap operator $U(\hat{\boldsymbol{q}}):{ }^{39}$

$$
\begin{aligned}
& \chi(t)=\left|\boldsymbol{\mu}_{e g}\right|^{2} \operatorname{Tr}\left[\rho_{g} e_{+}^{-\mathrm{i} \int_{0}^{t} U(\hat{\boldsymbol{q}}, \tau) \mathrm{d} \tau}\right] \\
& =\left|\boldsymbol{\mu}_{e g}\right|^{2} e^{-\mathrm{i} \omega_{e g}^{\mathrm{av}} t} \exp \left[-\sum_{n=2}^{\infty} g_{n}(t)\right],
\end{aligned}
$$

where $U(\hat{\boldsymbol{q}})=H_{e}(\hat{\boldsymbol{q}})-H_{g}(\hat{\boldsymbol{q}})$ is given by the difference between the Hamiltionians for the electronic ground and excited state, $\hat{\boldsymbol{q}}$ is the position operator of nuclear degrees of freedom, $\omega_{e g}^{\text {av }}=\operatorname{Tr}\left[\rho_{g} U(\hat{\boldsymbol{q}})\right]$ is the ground state thermal average of the electronic energy gap operator, $\rho_{g}$ is the equilibrium density operator and $g_{n}(t)$ is the $n$th order cumulant.

If the fluctuations of the energy operator follow Gaussian statistics, the above expression is exact at second order. Real systems do not generally follow Gaussian statistics, and truncation at second order yields the secondorder cumulant approximation. ${ }^{39,55}$ Even with truncation at second order, the cumulant method is able to approximately capture effects due to anharmonic degrees of freedom by mapping the energy gap fluctuations to a fictitious harmonic bath. ${ }^{38}$ The linear response function is then determined by the 2 nd order cumulant $g_{2}(t)$, which can be evaluated as $^{39}$

$$
\begin{aligned}
g_{2}(t)= & \int_{0}^{t} \mathrm{~d} \tau_{2} \int_{0}^{\tau_{2}} \mathrm{~d} \tau_{1} C_{\delta U}\left(\tau_{2}-\tau_{1}\right) \\
= & \frac{1}{\pi} \int_{0}^{\infty} \mathrm{d} \omega \frac{\mathcal{J}(\omega)}{\omega^{2}}\left[\operatorname{coth}\left(\frac{\beta \omega}{2}\right)[1-\cos (\omega t)\right. \\
& -\mathrm{i}[\sin (\omega t)-\omega t]]
\end{aligned}
$$

where $C_{\delta U}(t)=\operatorname{Tr}\left[\rho_{g} \delta U(\hat{\boldsymbol{q}}, t) \delta U(\hat{\boldsymbol{q}}, 0)\right]$ is the quantum autocorrelation function of energy gap fluctuations $\delta U(\hat{\boldsymbol{q}})=U(\hat{\boldsymbol{q}})-\omega_{e g}^{\mathrm{av}}$ and $\mathcal{J}(\omega)$ is the spectral density. The spectral density $\mathcal{J}(\omega)$ can be obtained directly from the quantum autocorrelation function $C_{\delta U}(t)$ via

$$
\mathcal{J}(\omega)=\mathrm{i} \theta(\omega) \int_{-\infty}^{\infty} \mathrm{d} t e^{\mathrm{i} \omega t} \operatorname{Im}\left\{C_{\delta U}(t)\right\}
$$

where $\theta(\omega)$ is the heaviside step function. However, $C_{\delta U}(t)$ is generally inaccessible for any realistic system and therefore a range of schemes have been developed to approximately construct $C_{\delta U}(t)$ from its purely classical counterpart $C_{\delta U}^{\mathrm{cl}}(t)$ using quantum correction factors. ${ }^{56-58} \mathrm{~A}$ standard choice for a quantum correction factor is the harmonic approximation, which can be derived by relating the quantum correlation function to its 
Kubo-transformed analogue that has the same symmetries as the classical correlation function. ${ }^{59,60}$ The harmonic quantum correction factor also guarantees that for a system with nuclear degrees of freedom described by a set of linearly coupled harmonic oscillators, the exact quantum correlation function can be reconstructed from its classical counterpart. ${ }^{38,61}$ Under this choice of quantum correction factor, the spectral density $\mathcal{J}(\omega)$ can be expressed as

$$
\mathcal{J}(\omega)=\theta(\omega) \frac{\beta \omega}{2} \int_{-\infty}^{\infty} \mathrm{d} t e^{\mathrm{i} \omega t} C_{\delta U}^{\mathrm{cl}}(t) .
$$

In practice, the classical autocorrelation function of the energy gap fluctuations can be obtained from computing vertical excitation energies along a molecular dynamics trajectory of the system propagated on the ground state potential energy surface (PES) ${ }^{41,42}$ In realistic systems, the second-order cumulant approach generally suffers from two sources of error: The truncation of the cumulant expansion at second order and the approximate reconstruction of a quantum correlation function from its purely classical counterpart. These different sources of error have been analyzed in detail for simple model systems. ${ }^{38}$

The cumulant method is particularly powerful in complex, condensed-phase systems with a large number of environmental degrees of freedom coupling to the optical excitations. The high frequency region of the spectral density showcases the couplings of high frequency vibrational modes of the chromophore to the energy gap and is responsible for the vibronic fine structure of the linear spectrum, whereas the low frequency region describes collective chromophore-environment modes that lead to spectral broadening. Other approaches to modeling linear optical spectroscopy ${ }^{37}$ do not describe both spectral contributions on the same footing. The ensemble approach ${ }^{19-24}$ samples uncorrelated chromophoreenvironment configurations and thus treats low frequency spectral broadening in a purely static way, ignoring any dynamic contributions from high frequency modes. The scheme does however allow for a fully atomistic representation of the environment, and previous studies have shown the need for large QM regions to fully describe the polarization between the chromophore and the electronic degrees of freedom of the environment that lead to spectral shifts. The Franck-Condon scheme $e^{25-32}$ allows for a dynamic, but usually harmonic, treatment of chromophore degrees of freedom described by the highfrequency region of the spectral density, but the approach does not generally incorporate an atomistic environment. Thus the Franck-Condon scheme does not account for any specific chromophore-environment polarization. In this work we assess how quantum mechanical polarization affects the spectral densities and linear spectra computed within the cumulant approach. We explicitly differentiate between static and dynamic coupling between the environment and the energy gap fluctuations. We describe coupling as static if it influences the low frequency

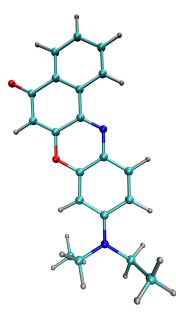

Nile Red

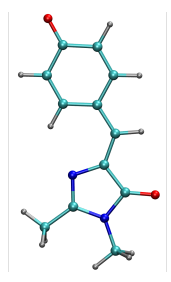

$\mathrm{HBDI}^{-}$

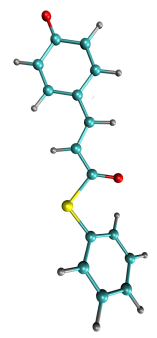

$\mathrm{pCT}^{-}$

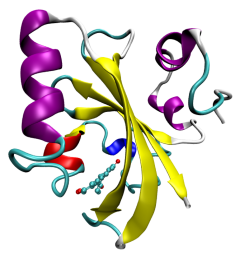

PYP
FIG. 1. The four systems considered in this work: The Nile red chromophore in different solvent environments; the deprotonated $p$-hydroxybenzylidene-2,3-dimethylimidazolinone $\left(\mathrm{HBDI}^{-}\right),{ }^{62,63}$ a GFP model chromophore, in water; the deprotonated trans-thiophenyl- $p$-coumarate $\left(\mathrm{pCT}^{-}\right),{ }^{64}$ a PYP model chromophore, in water; and the full photoactive yellow protein (PYP).

$\left(\omega<100 \mathrm{~cm}^{-1}\right)$ region of the spectral density, thus mainly affects the broadening of the spectrum. Dynamic coupling influences the high frequency region of the spectral density and thus directly alters the coupling of chromophore degrees of freedom to the electronic energy gap that are responsible for the vibronic fine-structure.

\section{B. Condensed phase systems}

To demonstrate the influence of the complex environment on computed spectral densities in realistic systems, we focus on three chromophores in different atomistic environments (See Fig. 1). We are interested in analyzing the coupling between the chromophore and its environment for a range of different solute-solvent interaction strengths and types, including both protic and aprotic, as well as polar and non-polar solvents. We first examine Nile red, a common solvatochromic dye, in cyclohexane, benzene, acetone, methanol, and water. Next, we focus on the deprotonated $p$-hydroxybenzylidene2,3-dimethylimidazolinone $\left(\mathrm{HBDI}^{-}\right)^{62,63}$ and transthiophenyl-p-coumarate $\left(\mathrm{pCT}^{-}\right)^{64}$ chromophores in water, which are model chromophores for green fluorescent protein (GFP) and photoactive yellow protein (PYP), respectively, and have been the focus of a series of experimental and theoretical studies in recent years. ${ }^{63-68}$ Finally, we study the behavior of photoactive yellow protein (PYP), a small pigment-protein complex that has been the focus of a detailed study on the influence of the QM treatment of the protein environment, ${ }^{19}$ where it was found that large QM regions of the protein environment are necessary in order to obtain converged excitation energies. Its relatively small size (1929 atoms) makes PYP an ideal model system to study how complex condensed phase environments couple to the dynamic energy gap fluctuations, which is of particular relevance for the study of energy transfer dynamics in pigment-protein complexes that rely on the computation of spectral densi- 


\begin{tabular}{c|c} 
System & Number of MM atoms \\
\hline Nile red in cyclohexane & 17736 \\
Nile red in benzene & 9762 \\
Nile red in acetone & 13332 \\
Nile red in methanol & 10428 \\
Nile red in water & 10608 \\
HBDI $^{-}$in water & 5340 \\
pCT $^{-}$in water & 5765 \\
PYP in water & 34209 \\
\hline
\end{tabular}

TABLE I. Number of atoms in the MM simulation cell.

ties to describe the system-bath coupling. Each of these systems has a single bright state of interest in the low energy region of the linear absorption spectrum.

\section{Computational details}

To generate trajectories from which to calculate classical correlation functions of energy gap fluctuations, we perform force field based classical MD simulations using the AMBER MD software package. ${ }^{69}$ For the Nile red chromophore we use the same force field as was used in our previous studies, ${ }^{23}$ which is based on the generalized AMBER force field (GAFF), ${ }^{70}$ but with the dihedral angle controlling rotations of the dimethylamino group around the $\mathrm{C}-\mathrm{N}$ bond reparameterized to match density-functional theory (DFT) calculations. The TIP3P model $^{71}$ is used for water and for all other solvents we use unmodified GAFF parameters. For HBDI ${ }^{-}$ in water, we use the same force field as in Ref. 36, which was again generated using GAFF parameters, but where the two dihedral angles controlling twists around the conjugated backbone were reparameterized using DFT calculations. For $\mathrm{pCT}^{-}$and PYP, we used the same force fields as described in Ref. 19.

Nile red, $\mathrm{pCT}^{-}$, and $\mathrm{HBDI}^{-}$were placed in large solvent boxes, with the total number of atoms in the MM simulation cells shown in Table I. A 20 ps temperature equilibration to $300 \mathrm{~K}$, followed by 400 ps of pressure equilibration in the the NPT ensemble was performed. This was followed by a 2 ns production calculation in the NVT ensemble of which the last 6 ps were extracted for computing the correlation function. The simulation time step was 2 fs and a Langevin thermostat with a collision frequency of $1 \mathrm{ps}^{-1}$ was used to keep the systems at a constant temperature. For PYP (PDB file: $1 \mathrm{NWZ}^{72}$ ), we took the equilibrated box of the protein solvated in TIP3P water from Ref. 19 and ran a 20 ps production calculation in the NVT ensemble at $300 \mathrm{~K}$. The same Langevin thermostat as for the other systems was used, but the MD time step was chosen to be 0.5 fs to match the parameters used in Ref. 19 for the equilibration. The last 6 ps of the production trajectory were extracted for the calculation of the classical autocorrelation functions.

From the 6 ps trajectories, snapshots were extracted every 2 fs, yielding a total of 3000 snapshots per system.

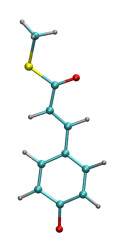

Region 1

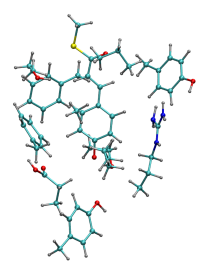

Region 2

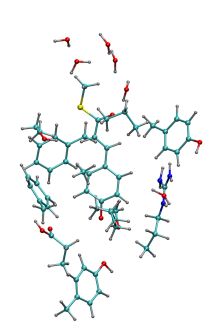

Region 3

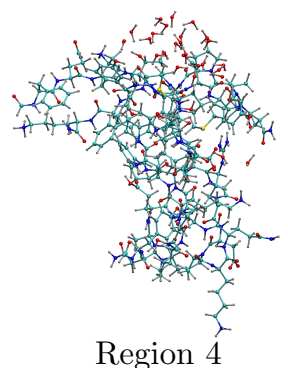

Region 4
FIG. 2. The four different QM regions used for calculating vertical excitation energies for photoactive yellow protein

For each of those snapshots, vertical excitation energies were computed for varying QM region sizes. For the chromophores in different solvents, QM regions were defined by placing spheres with a radius of $R_{\text {cut }}$ on each atom of the chromophore and including all solvent molecules with a center of mass within the volume defined by the overlapping spheres in the QM region. All solvent molecules in the MM box not within the QM region defined by a given choice of cutoff radius were included in the excitation energy calculation as fixed classical point charges, where the point charges were taken from the MM force field defining the solvent molecule. For Nile red, four different choices of cutoff radius, $R_{\text {cut }}=0 \AA$ (corresponding to a $\mathrm{QM}$ region only containing the chromophore), $R_{\text {cut }}=4 \AA, R_{\text {cut }}=6 \AA$ and $R_{\text {cut }}=8 \AA$ were used. For $\mathrm{HBDI}^{-}$and $\mathrm{pCT}^{-}$in water, QM region sizes were defined in the same way, but with $R_{\text {cut }}=0 \AA, R_{\text {cut }}=3 \AA$, $R_{\text {cut }}=6 \AA$ and $R_{\text {cut }}=8 \AA$ as the cutoff radius.

For PYP, the QM region in the calculation of vertical excitation energies can no longer be defined by a simple cutoff radius around the chromophore, as this would result in cutting through arbitrary covalent bonds of the protein environment. Instead, we consider the following four QM regions (see Fig. 2):

- QM region 1: Chromophore + C-S from the CYS69 residue (QM region charge: -1 )

- QM region 2: Same as region 1, plus H-bonded residues GLH-46 and TYR-42, as well as closeby residues ARG-52, VAL-66, THR-50, TYR-98, PHE-62, PHE-96, PRO-68, ALA-67 and THR-70 (QM region charge: 0 )

- QM region 3: Same as region 2, plus all water molecules with a center of mass that is within $4 \AA$ of any atom defined in QM region 1 (QM region charge: 0 )

- QM region 4: Residues 42-70 and 92-106 including the protein backbone as well as either C-O or $\mathrm{N}-\mathrm{H}$ atoms on adjacent residues to prevent a cut through the peptide bond. Additionally, all water molecules with centers of mass within $6 \AA$ of any atom in QM region 1 are also included in the $\mathrm{QM}$ region (QM region charge: 0 ). 


\begin{tabular}{|c|c|c|c|c|}
\hline \multicolumn{5}{|c|}{ Nile Red } \\
\hline & $0 \AA$ & $4 \AA$ & $6 \AA$ & $8 \AA$ \\
\hline Cyclohexane & 42 & 122 & 421 & 609 \\
\hline Benzene & 42 & 136 & 339 & 549 \\
\hline Acetone & 42 & 152 & 333 & 588 \\
\hline Methanol & 42 & 194 & 338 & 674 \\
\hline Water & 42 & 191 & 387 & 708 \\
\hline \multicolumn{5}{|c|}{ GFP and PYP model chromophores in water } \\
\hline & $0 \AA$ & $3 \AA$ & $6 \AA$ & $8 \AA$ \\
\hline $\mathrm{HBDI}^{-}$ & 27 & 75 & 299 & 578 \\
\hline $\mathrm{pCT}^{-}$ & 29 & 75 & 337 & 633 \\
\hline \multicolumn{5}{|c|}{ Photoactive Yellow Protein } \\
\hline & Region & egion & egion & egion \\
\hline & 22 & 159 & 175 & 784 \\
\hline
\end{tabular}

TABLE II. Average number of QM atoms in the calculation of the vertical excitation energies used to construct the energy gap auto-correlation function.

In places where covalent bonds are cut in the definition of the QM regions, the QM region atoms were capped with hydrogens and the MM charges were adjusted using the QM/MM functionality ${ }^{73}$ within the AMBER code. This QM region partitioning is roughly consistent with that from Ref. 19, but other QM partitioning methods ${ }^{74-76}$ may provide improved results. The average number of atoms contained in all QM regions is shown in Table II.

To construct the energy gap correlation functions, we computed vertical excitation energies for all 3000 snapshots of the 6 ps trajectories for all QM region sizes. All excited state calculations were performed using time-dependent DFT (TDDFT) in the Tamm-Dancoff approximation $^{77}$ and the CAM-B3LYP ${ }^{78}$ functional as implemented in the GPU-accelerated TeraChem code. ${ }^{46}$ For the Nile red calculations, a $6-31 \mathrm{G}^{*}$ basis set ${ }^{79}$ was used throughout. Although this basis set is too small to yield fully converged vertical excitation energies, previous work ${ }^{68}$ has shown that it captures the correct distribution of an ensemble spectrum of vertical excitations. For calculations involving $\mathrm{HBDI}^{-}$, a larger 6$31+\mathrm{G}^{*}$ basis set ${ }^{79}$ is used to better capture the more delocalized electronic excited state of the anion, ${ }^{65}$ in line with previous studies focused on modeling its absorption spectrum. ${ }^{68}$ For $\mathrm{pCT}^{-}$in water and in its protein environment, we again use the $6-31 \mathrm{G}^{*}$ basis set, which allows us to include the entire protein in the QM region with the same quality basis set as the chromophore.

The vertical excitation energies computed for the 6 ps trajectories for all systems and QM regions are then used to construct energy gap autocorrelation functions, which, after a Fourier transform and the application of the harmonic quantum correction factor, yield the spectral densities. To ensure well-behaved Fourier transforms, a decaying exponential of the form $\exp (-|t| / \tau)$ is applied to all classical correlation functions $C_{\delta U}^{\mathrm{cl}}(t)$, where $\tau=500 \mathrm{fs}$ is chosen for all systems, corresponding to a Lorentzian broadening of all peaks in the spectral density. Linear spectra were then computed according to Eqs. 1 and 2. Because the Condon approximation is only approximately valid in realistic systems, the square of the transition dipole moment in Eqn. 2 is replaced by its average value computed over all 3000 snapshots for a given QM region.

The relatively short 6 ps trajectories are not sufficient to fully converge spectral densities and linear spectra, which would require the correct sampling of both the momentum distribution of high-frequency chromophore degrees of freedom and low frequency collective environment degrees of freedom responsible for static broadening. The fixed MM point charges provide polarization of the QM ground state electron density, but the QM environment provides mutual polarization of the chromophore and the environment, as well as polarization of the transition density. The large QM region also intrinsically accounts for charge transfer between the chromophore and the environment as part of what we term QM polarization. Here we specifically focus on how this QM polarization influences both low- and high-frequency features in the spectral density and the resulting linear absorption spectrum.

\section{RESULTS}

\section{A. Weak dynamic coupling: Nile red in various solvents}

We investigate the dependence of the spectral density and linear spectrum with QM region size for Nile red in five different solvents: cyclohexane, benzene, acetone, methanol, and water. Cyclohexane and benzene are both non-polar solvents, but previous studies show that some solute-solvent interaction occurs between benzene and Nile red in the form of $\pi$-stacking. ${ }^{23}$ Acetone, methanol, and water are polar solvents, but acetone is aprotic and expected to only weakly interact with hydrogen-bonding sites of Nile red, whereas methanol and water are expected to form hydrogen bonds with the chromophore. Thus the five solvent environments chosen cover both strong and weak solvent polarity effects, as well as strong and weak solute-solvent interactions.

Fig. 3 shows the spectral densities computed for Nile red in benzene and in water, where different amounts of the environment are treated at the QM level when computing vertical excitation energies. The result for all other solvents can be found in SI section II. For both Nile red in water and in benzene, the spectral density consists of a continuous low frequency region that we ascribe to solvent degrees of freedom and slow collective solventchromophore motion coupling to the chromophore excitation energy, and sharp, high-frequency peaks that we ascribe to vibrational modes of the chromophore. The vibrational frequencies in the spectral density are not expected to precisely match the frequencies that would be obtained by performing a normal mode analysis of the chromophore in vacuum or implicit solvent. The rea- 


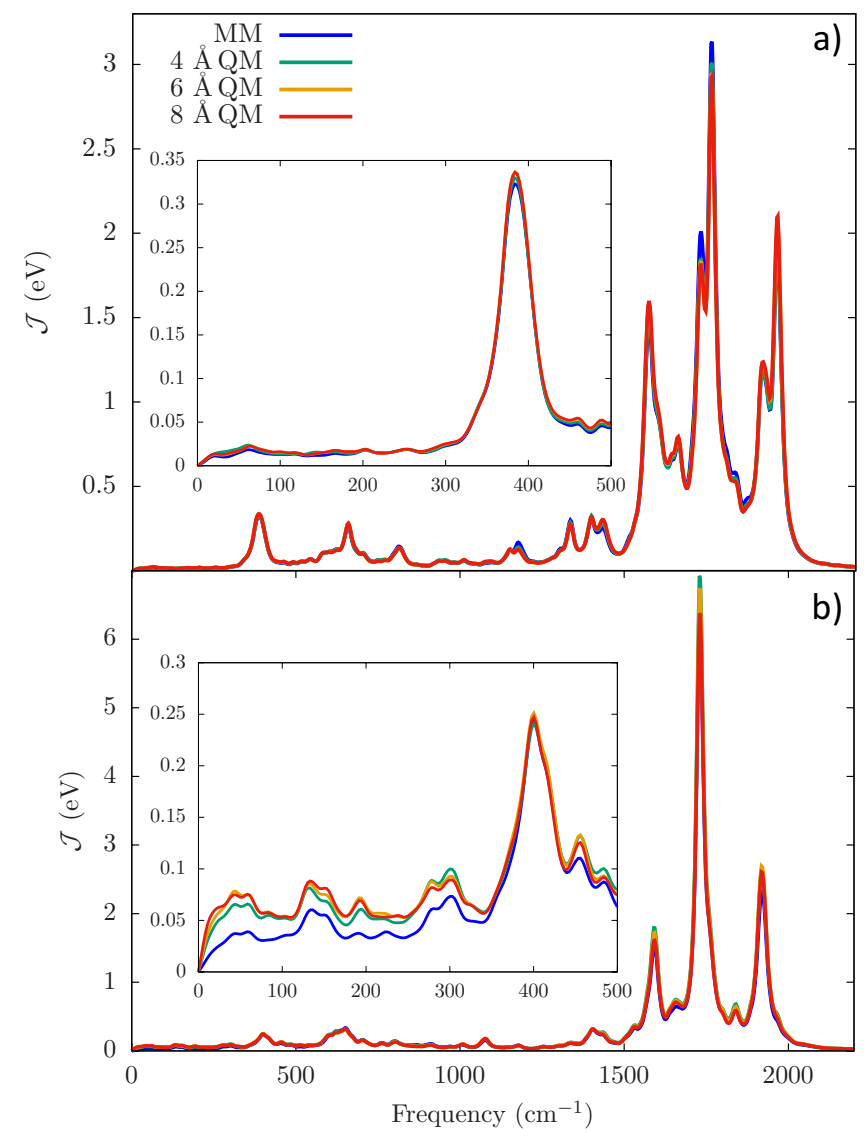

FIG. 3. Spectral density of Nile red in a) benzene and b) water for varying sizes of the QM region. The inset shows a zoom-in of the low frequency region.

son for this is that the full, generally anharmonic, potential energy surface of both chromophore and solvent degrees of freedom is sampled in the cumulant approach and anharmonic effects, ${ }^{38}$ as well as the influence of direct solute-solvent coupling, are encoded in the spectral density.

From Fig. 3, it is clear that the QM polarization of the environment has a minor impact on the high frequency features of the spectral density for Nile red, with a pure MM representation of the environment producing almost identical results as a full $8 \AA \mathrm{QM}$ region. For Nile red in benzene, the low-frequency part of the spectral density up to about $300 \mathrm{~cm}^{-1}$ that is dominated by slow chromophore and solvent degrees of freedom is also invariant with respect to the QM environment. By computing the effective reorganization energies of the system-bath coupling for the spectral densities with and without QM polarization, we find that an MM treatment of the environment misses only $2 \%$ of the reorganization energy (See SI Sec. III). However, for Nile red in water, the larger QM regions increase the amount of spectral weight in the low-frequency region of the spectral density. For water as the solvent, an MM treatment of the environment misses about $25 \%$ of the reorganization energy, and
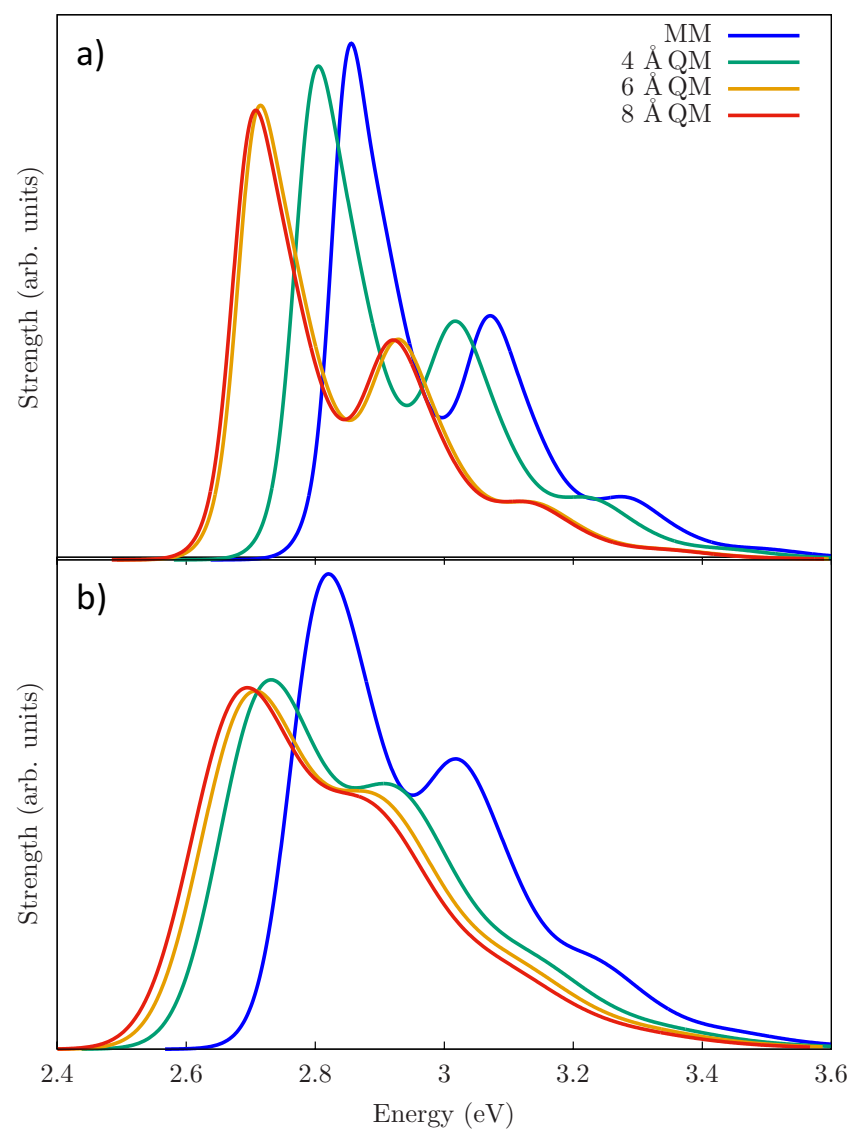

FIG. 4. Linear absorption spectra of Nile red in a) benzene and b) water as computed from the spectral density for varying sizes of the QM region.

nearly all contributions added by the QM solvent environment are confined to the $\omega<100 \mathrm{~cm}^{-1}$ region of the spectral density (See SI Sec. III). We ascribe this larger reorganization energy to the much stronger solute-solvent interactions for Nile red in water that are better described by a QM representation of the solvent environment.

Fig. 4 shows the linear spectra computed from the spectral densities of Nile red in benzene and water for all QM region sizes. For Nile red in benzene, the linear spectrum shows clear vibronic fine structure, with a main peak and two smaller peaks of decreasing intensity. Increasing the QM region red-shifts the linear spectrum, in line with results obtained for spectra computed in the ensemble approach in a previous study. ${ }^{23}$ The shift of the average excitation energy upon including QM polarization is in line with red-shifts commonly observed when computing ensemble spectra in explicit solvent environments ${ }^{23,37,52}$ However, despite the energetic red-shift, the overall shape of the linear spectrum is essentially unaltered by the QM environment, in agreement with the very minor changes observed in the spectral density. For Nile red in water, the linear spectrum shows a similar overall red-shift with respect to increasing the QM region size, but there are also differences in 
spectral shape. The spectrum computed in a pure MM environment shows well-defined vibronic peaks, whereas the larger QM regions produce a broader spectrum with smoother vibronic features. This behavior can be attributed to the fact that the high frequency peaks in the spectral density for Nile red in water that are responsible for the vibronic fine structure of the spectrum are nearly unaltered by the QM polarization, and thus are not sensitive to the dynamic coupling provided by the solvent environment. In contrast, the low frequency region of the spectral density, which is due to collective solvent motion and is responsible for the static broadening of the spectrum, is enhanced with the larger QM regions.

We also computed the linear absorption spectra for Nile red in cyclohexane, acetone, and methanol, with the results shown in the SI, section II. For all solvent environments, the high frequency modes of the spectral density are insensitive to the QM polarization of the solvent and, for all solvents apart from water, the low frequency region of the spectral density also shows little dependence on the computational treatment of the environment. All linear spectra red-shift upon increasing the amount of the environment that is treated as QM due to excited state polarization. However, the overall shape of the spectra remains relatively unaltered, apart from the extra broadening observed for Nile red in water, supporting that Nile red is insensitive to dynamic coupling of the QM environment to energy gap fluctuations. Although the spectral shapes of Nile red in solvents apart from water is nearly invariant with respect to the QM polarization of the environment, there are clear differences between the linear spectra in different solvents. The spectrum in cyclohexane shows the largest degree of vibronic fine structure, which we ascribe to the weak solute-solvent interaction leading to small spectral weight in the low frequency region of the spectral density and therefore a small degree of solvent broadening. Even though benzene is a non-polar and acetone is a polar solvent, the increased solute-solvent interaction strength in benzene due to $\pi$-stacking ${ }^{23}$ leads to comparable solvent broadening. Methanol, a polar solvent forming hydrogen bonds, leads to a broadened spectral shape more closely resembling that of Nile red in water. We note again that due to the short 6 ps trajectory, these spectra are missing the additional static broadening that would arise from computing the energy-gap correlation function over longer timescales.

Our calculations demonstrate that for Nile red in less strongly interacting solvents (i.e., not water), cumulantbased spectra obtained by computing energy gap correlation functions with the entire environment treated as classical point charges will likely agree in shape with those computed with a QM environment. The red-shift of the spectrum due to increasing QM region size can be estimated from the average red-shift computed from a smaller number of snapshots, dramatically reducing the computational cost associated with a full QM treatment of the environment in the cumulant approach.

\section{B. Strong dynamic coupling: $\mathrm{HBDI}^{-}$and $\mathrm{pCT}^{-}$in water}

Fig. 5 shows the spectral density computed for the GFP and PYP model chromophore anions in water, again computed for a 6 ps trajectory and different sizes of the QM region. As with Nile red in water, the low frequency regions of the spectra show enhancement when going from classical point charges to QM polarization of the environment. This increased static polarization can be quantified by computing the cumulative difference in reorganization energy between the spectral density computed with the large QM environment and the spectral density computed for MM point charges, which shows a sharp rise for frequencies below $100 \mathrm{~cm}^{-1}$ (See SI Sec. III). Similar to Nile red in water, the MM solvent environment misses a significant amount of reorganization energy contained in the spectral density constructed with the full QM solvent environment $\left(21 \%\right.$ for $\mathrm{HBDI}^{-}$ and $32 \%$ for $\mathrm{pCT}^{-}$), with most of the contributions originating from the low frequency $\left(\omega<200 \mathrm{~cm}^{-1}\right)$ region of the spectral density. However, unlike for Nile red, both $\mathrm{HBDI}^{-}$and $\mathrm{pCT}^{-}$in water also show a significant redistribution of spectral weight in the high-frequency $\left(\omega>1000 \mathrm{~cm}^{-1}\right)$ region of the spectral density in going from MM to QM solvent. For example, a peak at $1370 \mathrm{~cm}^{-1}$ for $\mathrm{HBDI}^{-}$loses more than half of its intensity when comparing the MM environment to an $8 \AA \mathrm{QM}$ region, whereas the peak at $1600 \mathrm{~cm}^{-1}$ is enhanced with the QM environment. The intensity changes for these high-frequency peaks are systematic with increasing QM region size, suggesting a cumulative dynamic polarization effect. The significant change in high-frequency peaks of the spectral density is not accompanied by a change in character of the excitation, as is evident from plots of the electron-hole density of the $\mathrm{S}_{1}$ state both in $\mathrm{MM}$ and in explicit QM solvent in Fig. 5.

It is difficult to rationalize the change in spectral density peak heights with a simple Franck-Condon picture where the state with larger charge separation is more stabilized by the QM environment and thus changes the Franck-Condon overlap integrals. The complication is that the fixed MM point charges provide polarization of the QM electron density in the ground state, and have in fact been found to over-polarize QM regions. ${ }^{80-83}$ These fixed charges, however, do not enter the TDDFT linear response equations and therefore do not screen the electron or hole density of the transition. This screening can be provided by QM polarization of the solvent or can be approximated using polarizable implicit solvent models.

To better understand the intensity changes in the highfrequency peaks of the spectral density, we compute the bond length time-correlation function and compare its Fourier transform to the spectral density. A detailed analysis aimed at identifying the origin of the highfrequency peaks in the spectral density for both $\mathrm{HBDI}^{-}$ and $\mathrm{pCT}^{-}$can be found in SI Sec. VI. From this analysis, we find that the features in the spectral densities of both $\mathrm{HBDI}^{-}$and $\mathrm{pCT}^{-}$that decrease in intensity with 

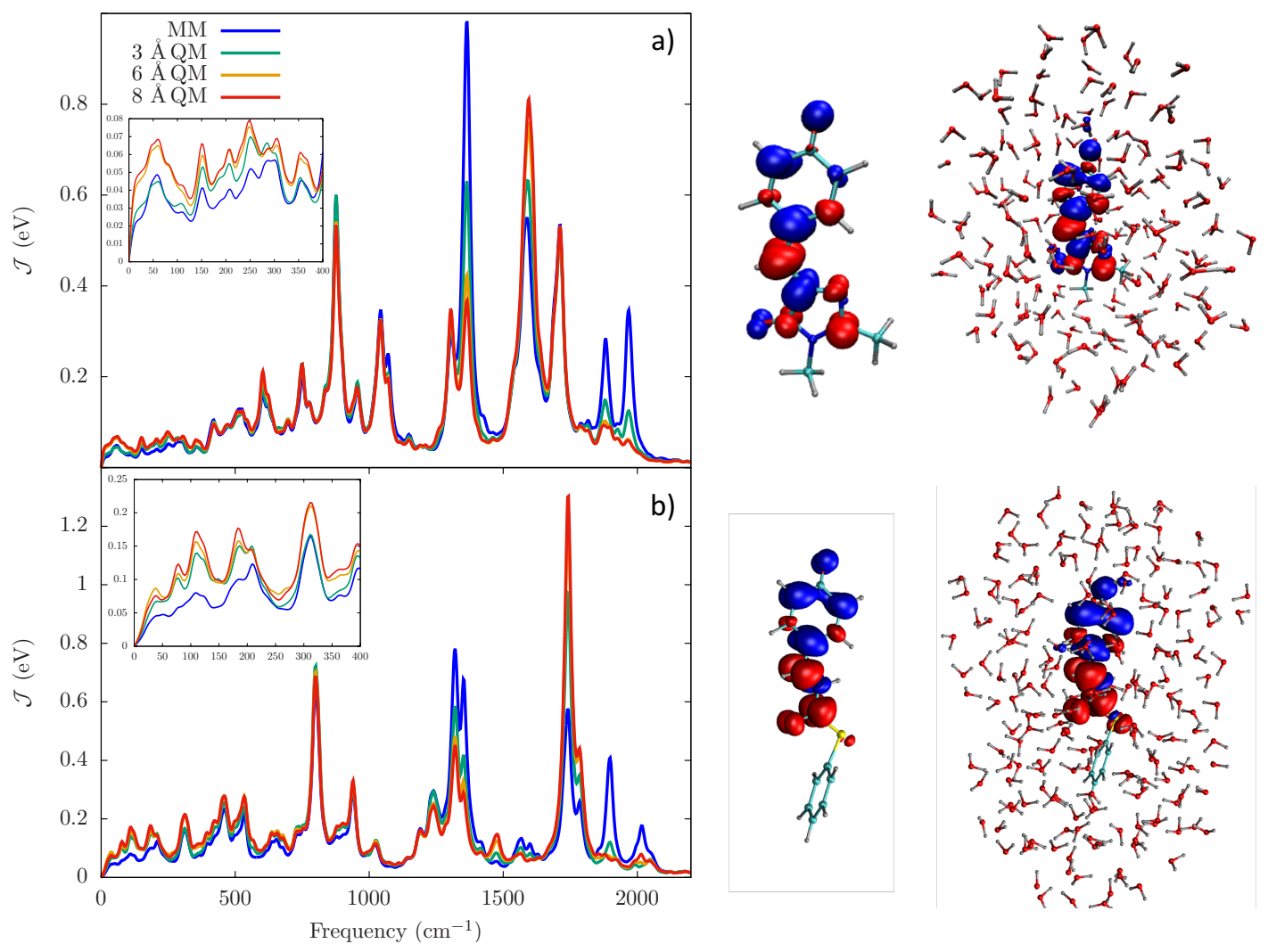

FIG. 5. Spectral density of a) $\mathrm{HBDI}^{-}$b) $\mathrm{pCT}^{-}$in water for varying sizes of the QM region. The inset shows a zoom-in of the low frequency region. Also shown are plots of the electron (red)-hole (blue) difference densities of the $\mathrm{S}_{1}$ state, as computed for a pure MM solvent environment and for an $8 \AA$ QM region.

increasing size of $\mathrm{QM}$ region all involve the conjugated carbon backbone connecting the phenolate (hole) to either the carbonyl for $\mathrm{pCT}^{-}$or the imidazole for $\mathrm{HBDI}^{-}$ (electron), see the density difference plots in Fig. 5. Due to the intramolecular electron-hole separation, any vibration coupling the electron and the hole is expected to strongly influence the energy gap fluctuations. However, as seen in the significant decrease of the transition dipole moment with increasing QM region size (see SI, section $\mathrm{V})$, the QM polarization screens the electron from the hole, decreasing the coupling of the $\mathrm{C}-\mathrm{C}$ vibrations to the optical excitation. Our analysis also shows spectral density peaks have increased intensity if the environment is treated at the QM level if they are due to vibrational modes that involve either the electron or the hole but not the conjugated bridge connecting the two. An example is the spectral peak at $1720 \mathrm{~cm}^{-1}$ for $\mathrm{pCT}^{-}$, which mainly involves the $\mathrm{C}-\mathrm{O}$ stretching mode of the carbonyl group. This finding also explains why the dependence of high frequency modes in the spectral density on $\mathrm{QM}$ region size is weaker in Nile red than in $\mathrm{pCT}^{-}$and $\mathrm{HBDI}^{-}$. Although the bright $\mathrm{S}_{1}$ state of Nile red has charge transfer character (See SI Sec. I), there is not a single conjugated bridge connecting the electron and the hole, and the vibrations responsible for individual peaks in the spectral density are generally more delocalized (See SI Sec. VII) and therefore do not couple strongly to solvent polarization.

The linear absorption spectra generated by the spectral densities for both systems are shown in Fig. 6. Due to the weight in low frequency regions of the spectral density in both systems, caused by strong solute-solvent interactions, both MM and QM environments produce relatively smooth spectra. In agreement with the results obtained for Nile red in water, the QM solvent environment leads to a red-shift of the absorption spectra and increased broadening due to the enhancement of the low frequency region of the spectral density. This low frequency contribution masks the vibronic fine structure, but if we compute the linear spectra for both $\mathrm{HBDI}^{-}$ and $\mathrm{pCT}^{-}$for the high frequency regions of the spectral density only (see the SI section IV), we find that due to the strong coupling of the solvent environment to dynamic features in the spectral density, the underlying vibronic fine structure of the linear spectra is also altered with QM polarization. The QM polarization provides enhancement of the vibronic tail of the linear spectrum for $\mathrm{pCT}^{-}$in water, which we ascribe to the redistribution of spectral weight from the region just below $1500 \mathrm{~cm}^{-1}$ to higher frequency modes in the spectral density. 


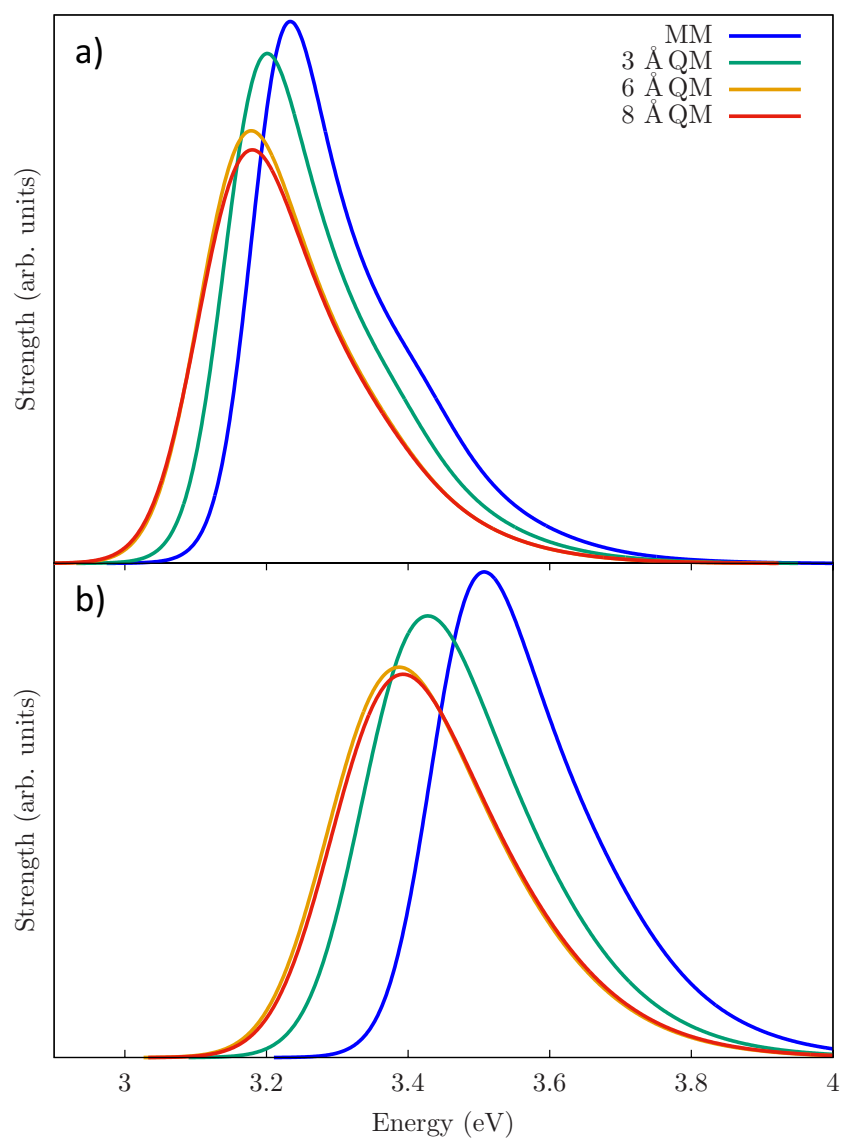

FIG. 6. Linear absorption spectra of a) $\mathrm{HBDI}^{-}$and b) $\mathrm{pCT}^{-}$ in water as computed for varying sizes of the $\mathrm{QM}$ region.

\section{Complex environmental interactions: Photoactive yellow protein}

We lastly focus on PYP as an example of a chromophore embedded in a protein environment that will likely be representative of pigment-protein complexes. As discussed in Sec. II C, the QM region in this system can no longer be defined through a simple cutoff radius but instead different nearby residues of the protein environment are included in the TDDFT calculations. The spectral densities computed for the four different QM regions considered are reported in Fig. 7. Similarly to $\mathrm{HBDI}^{-}$ and $\mathrm{pCT}^{-}$in water, both the high and low frequency regions of the spectral density show a strong dependence on QM region size. However, unlike for the two chromophores in water, there is no systematic trend with respect to increasing the QM region size. Certain high frequency peaks, such as the main peaks at $1350 \mathrm{~cm}^{-1}$ and $1580 \mathrm{~cm}^{-1}$, initially show a large decrease in intensity going from QM region 1 (containing only the chromophore) to $\mathrm{QM}$ region 2 (containing the chromophore and all residues interacting with it through hydrogen bonding and $\pi$-stacking) and QM region 3 (same as QM region 2 , but also including nearby water molecules). However, if the size of the QM region is further increased to in-

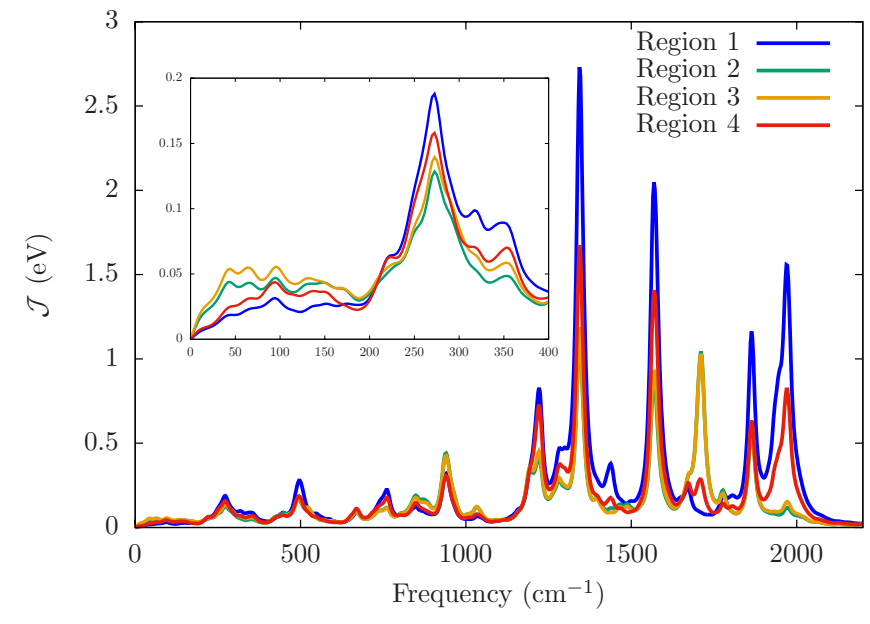

FIG. 7. Spectral density of the Photoactive Yellow Protein for varying sizes of the QM region. The inset shows a zoom-in of the low frequency region.

clude almost half of the protein, the intensity increases. The reduction of most of the high frequency peaks in the spectral density is the reason why the total reorganization energy for PYP decreases for the largest QM region (by $18 \%$, see SI Sec. III), in contrast to all other systems studied in this work. Unlike for Nile red in all solvents or $\mathrm{pCT}^{-}$and $\mathrm{HBDI}^{-}$in water, where most of the discrepancy in reorganization energy between an MM and a QM environment was confined to the low frequency region of the spectral density, for PYP these changes are almost exclusively due to high frequency modes above $1000 \mathrm{~cm}^{-1}$ (see SI Sec. III).

To understand the non-systematic nature of the changes of the spectral density with respect to QM region size, we again analyze both the average transition dipole moment of the $\mathrm{S}_{1}$ excited state and the origin of individual high frequency peaks in the spectral density. A graph of the computed transition dipole moments for different $\mathrm{QM}$ regions can be found in SI Sec. V, whereas the peak assignment of the spectral density is carried out in SI Sec. VI. The reason for the non-systematic convergence behavior of high frequency peaks in the spectral density with increasing QM region sizes can be traced to inconsistent amount of screening as seen by changes in the transition dipole moment. Changing from a pure MM representation of the protein environment to QM region 2 leads to a large drop in the transition dipole moment of about 1.5 D, much larger than for any of the other systems studied in this work. Increasing the QM region size to $\mathrm{QM}$ region 4 increases the transition dipole moment by about $0.5 \mathrm{D}$, suggesting that the medium sized QM regions 2 and 3 over-screen the interaction between the electron and hole.

The linear spectra of PYP obtained for all four QM regions, in comparison with the experimental spectrum, ${ }^{84}$ can be found in Fig. 8. All linear spectra show evidence of vibronic fine structure, consisting of a main peak and 


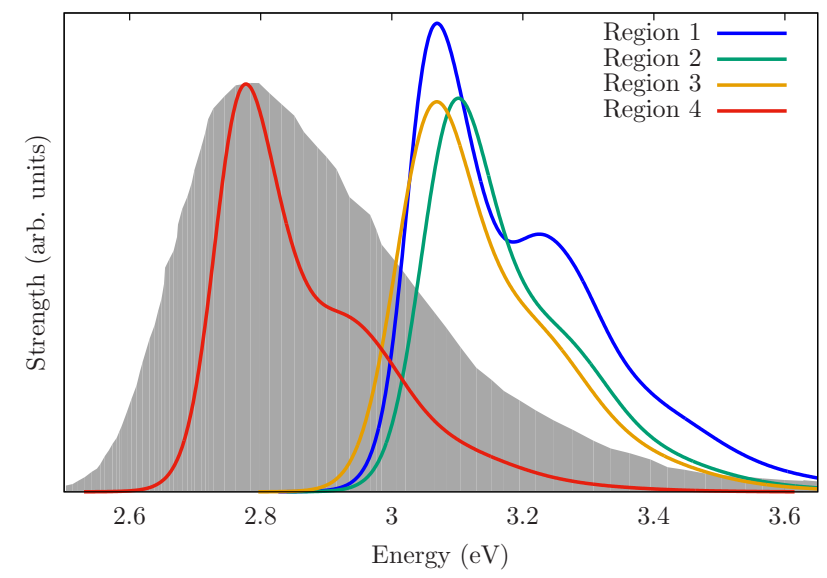

FIG. 8. Linear absorption spectra of photoactive yellow protein computed within the cumulant approach for varying sizes of the QM region. The experimental absorption spectrum ${ }^{84}$ is shown in gray and all simulated spectra are red-shifted by $0.18 \mathrm{eV}$ such that the absorption maximum of the simulated spectrum for QM region 4 agrees with the experimental spectrum.

a shoulder at higher energy. For the MM protein environment, the shoulder is very pronounced, but almost disappears completely for QM regions 2 and 3, before becoming evident again for the largest $\mathrm{QM}$ region 4 . The intensity of the shoulder is directly related to the strength with which high frequency vibrational modes couple to the energy gap, and thus directly follows the changes in spectral weight of the high frequency region of the spectral density with changing QM region (See Sec. III of the SI for a comparison of the contributions of high frequency regions to the spectral density for different QM regions). Furthermore, the linear spectrum red-shifts with increasing QM region but the shift is no longer systematic. QM regions 2 or 3 show almost no shift going from MM to QM environment, whereas for the largest QM region, the linear spectrum red-shifts by more than $0.3 \mathrm{eV}$. A similar behavior of the spectral shift with QM region was previously observed for this system with spectra computed in a classical ensemble approach. ${ }^{19}$ However, with the cumulant based approach, the shape of the absorption spectrum is significantly improved over the ensemble approach computation from Ref. 19, which lacks dynamic spectral contributions.

The spectra computed with the cumulant approach shown in Fig. 8 are all significantly too narrow compared to the experimental spectrum. This can be partially ascribed to the relatively short 6 ps trajectory used to compute the energy gap correlation functions, which must necessarily fail in correctly capturing static broadening and low frequency collective modes in the protein environment. To approximately account for static broadening effects in the absorption lineshape,${ }^{85}$ we performed a longer $1.2 \mathrm{~ns} \mathrm{MD}$ simulation and computed average energy gaps for 6 ps segments of that trajectory (see
Sec. VIII of the SI for further details). A similar strategy has been successfully applied recently to the modelling of the absorption lineshape of a chromophore embedded in the intercalation pocket of DNA. ${ }^{41}$ The additional static broadening computed for this longer $1.2 \mathrm{~ns}$ trajectory is insufficient to produce the correct spectral width for all QM regions.

One source of error in our calculations is the use of MM dynamics from which to compute the spectral densities, where the mismatch between the MM potential describing the dynamics and the DFT/TDDFT potential describing the energy gap fluctuations likely leads to poor representation of the high frequency contributions to the spectral density. $8,9,40,86-88$ Previous work that compared MM MD and QM/MM ab initio MD for this system also demonstrated that including protein residues hydrogenbonded to the chromophore in the QM region significantly influences the chromophore dynamics. ${ }^{19}$ Furthermore, it should be noted that a 6 ps trajectory is likely too short to sample the correct momentum distribution for the high frequency vibrational modes, such that the heights of peaks in the spectral density responsible for the asymmetry of the spectrum are not fully converged. The asymmetric shape of the ensemble spectrum for this system also suggests that the energy gap fluctuations are non-Gaussian, meaning that third-order cumulant contributions could be significant. ${ }^{38}$ Finally, our previous work on the PYP chromophore in water has demonstrated that nuclear quantum effects can have a significant influence on computed ensemble spectra, ${ }^{68}$ and it is possible that these effects play a role in the protein as well, especially given the strong hydrogen bonds between the phenolate oxygen of the chromophore and the GLH- 46 and TYR-42 residues present in the protein structure. ${ }^{89}$

\section{CONCLUSION}

Calculating accurate spectral densities is important for excitation energy transfer dynamics in multichromophore systems, as well as for condensed phase linear and nonlinear spectroscopy. Simulating linear spectra via the cumulant approach offers a variety of advantages over standard techniques, such as capturing the effects of anharmonic PESs not captured by the FranckCondon approach, improving spectral lineshapes over the ensemble approach from the presence of vibronic features, and not applying a separation of timescales for the chromophore and environment degrees of freedom. These advantages come at a steep computational cost and the cumulant approach has only recently been applied to the computation of linear absorption spectra. Here we analyze spectral densities and linear absorption spectra computed via the cumulant approach with increasing amounts of QM polarization of the environment. For Nile red we found that, because of the delocalized nature of the chromophore vibrations, the high frequency region of the spectral density was not sensitive to the polarization 
provided by the QM solvent environment. This weak dynamic coupling yields a similar shape for the absorption spectrum when the solvent environment is treated with MM point charges or treated with a large QM region, opening the door to a large reduction in computational cost by using MM charges for the environment. For $\mathrm{HBDI}^{-}$and $\mathrm{pCT}^{-}$in water, we found that both the chromophore carbon backbone vibration that strongly couples the electron and hole and isolated vibrations for the electron or hole were quite sensitive to QM region size, showing strong dynamic coupling to the polarizable environment with high-frequency peaks in the spectral density either increasing or decreasing in intensity with a larger QM region. For these systems, we find that the QM region sizes necessary to reach converged spectral densities are at least a full solvation shell, comparable to the sizes necessary to converge ensemble spectra in previous studies, requiring large computational effort to capture the full effect of polarization on the spectral density. For these two systems, the MM environment not only leads to a blue shift of the spectra in these systems, but also underestimates the total reorganization energy computed from the spectral density by up to $32 \%$. Most of the underestimation of the reorganization energy is contained in the low frequency region of the spectral density, leading to an underestimation of the static broadening of the computed spectra. For $\mathrm{HBDI}^{-}$and $\mathrm{pCT}^{-}$in water the redistribution of spectral weight in the high frequency region of the spectral density with QM polarization additionally alters the vibronic fine structure.

For solvated dyes, the convergence behavior of the spectral density and the linear absorption spectrum with increasing QM region size is systematic. For photoactive yellow protein however, the convergence behavior with respect to the size of the QM region is not systematic, mirroring results obtained for absorption spectra computed in the ensemble approach in a previous study. We demonstrate that when computing the spectral density of system-bath coupling for chromophores embedded in complex protein environments, the size of the QM region has to be carefully chosen in order to avoid an over or underestimation of environmental polarization effects. In contrast to the other systems, we find that for PYP the use of an MM environment underestimates the reorganization energy compared to the large QM region, by $18 \%$ , due mostly to the changes to the high frequency region of the spectral density. Our results suggest that for photoactive yellow protein, there is strong dynamic coupling with the environment and a large QM region is necessary to capture the full vibronic response.

Although the results presented in this work focus on the calculation of linear absorption spectra via the cumulant approach, spectral densities constructed from classical correlation functions of energy gap fluctuations are an integral part of first-principles simulations of nonlinear spectroscopy and the exciton dynamics of multichromophore pigment-protein complexes. In linear spectra, the influence of the rescaling of high frequency peaks in the spectral density due to QM polarization of the environment is largely masked by the effect of low frequency static broadening. However, nonlinear spectra will be considerably more sensitive to the coupling between fast chromophore vibrations and environmental polarization. Because the spectral density is the only input needed for calculation of nonlinear spectroscopy within the cumulant framework, our conclusions will carry over directly to the modeling of ultrafast energy relaxation of molecules in the condensed phase.

\section{ACKNOWLEDGMENTS}

This work was supported by the Department of Energy CTC and CPIMS programs (Grant No DE-SC0019053). L.S. also acknowledges the support from the University of California Merced start-up funding.

${ }^{1}$ M. Wendling, M. A. Przyjalgowski, D. Gülen, S. I. E. Vulto, T. J. Aartsma, and R. van Gondelle, "The quantitative relationship between structure and polarized spectroscopy in the FMO complex of Prosthecochloris aestuarii: refining experiments and simulations," Photosynth. Res. 71, 99-123 (2002).

${ }^{2}$ J. Adolphs and T. Renger, "How Proteins Trigger Excitation Energy Transfer in the FMO Complex of Green Sulfur Bacteria," Biophys. J. 91, 2778-2797 (2006).

${ }^{3}$ C. Olbrich, J. Strümpfer, K. Schulten, and U. Kleinekathöfer, "Theory and Simulation of the Environmental Effects on FMO Electronic Transitions," J. Phys. Chem. Lett. 14, 1771-1776 (2011).

${ }^{4}$ C. Kreisbeck and T. Kramer, "Long-Lived Electronic Coherence in Dissipative Exciton Dynamics of Light-Harvesting Complexes," J. Phys. Chem. Lett. 3, 2828-2833 (2012).

${ }^{5}$ S. Shim, P. Rebentrost, S. Valleau, and A. Aspuru-Guzik, "Atomistic Study of the Long-Lived Quantum Coherences in the Fenna-Matthews-Olson Complex," Biophys. J. 102, 649-660 (2012).

${ }^{6}$ D. J. Cole, A. W. Chin, N. D. M. Hine, P. D. Haynes, and M. C. Payne, "Toward ab initio optical spectroscopy of the fennamatthews-olson complex," J. Phys. Chem. Lett. 4, 4206-4212 (2013).

${ }^{7}$ L. Viani, M. Corbella, C. Curutchet, E. J. O'Reilly, A. OlayaCastro, and B. Mennucci, "Molecular basis of the excitonphonon interactions in the PE545 light-harvesting complex," Phys. Chem. Chem. Phys. 16, 16302 (2014).

${ }^{8}$ S. Chandrasekaran, M. Aghtar, S. Valleau, A. Aspuru-Guzik, and U. Kleinekathöfer, "Influence of Force Fields and Qauntum Chemistry Approach on Spectral Densities of BChl $a$ in Solution and in FMO Proteins," J. Phys. Chem. B 119, 9995-10004 (2015).

${ }^{9}$ M. K. Lee and D. F. Coker, "Modeling Electronic-Nuclear Interactions for Excitation Energy Transfer Processes in LightHarvesting Complexes," J. Phys. Chem. Lett. 7, 3171-3178 (2016).

${ }^{10}$ M. K. Lee, P. Huo, and D. F. Coker, "Semiclassical Path Integral Dynamics: Photosynthetic Energy Transfer with Realistic Environment Interactions," Annu. Rev. Phys. Chem. 67, 639668 (2016).

${ }^{11}$ M. K. Lee, K. B. Bravaya, and D. F. Coker, "First-Principles Models for Biological Light-Harvesting: Phycobiliprotein Complexes from Cryptophyte Algae," J. Am. Chem. Soc. 139, 78031814 (2017).

${ }^{12}$ S. M. Blau, I. G. Bennett, C. Kreisbeck, G. D. Scholes, and A. Aspuru-Guzik, "Local protein solvation drives direct downconversion in phycobiliprotein PC645 via incoherent vibronic transport," PNAS 115, E3342-E3350 (2018). 
${ }^{13}$ M. I. Mallus, Y. Shakya, J. D. Prajapati, and U. Kleinekathöfer, "Environmental effects on the dynamics in the light-harvesting complexes LH2 and LH3 based on molecular simulations," Chem. Phys. 515, 141-151 (2018).

${ }^{14} \mathrm{~S}$. J. Jang and B. Mennucci, "Delocalized excitons in natural light-harvesting complexes," Rev. Mod. Phys. 90, 035003 (2018).

${ }^{15}$ J. C. Dean and G. D. Scholes, "Coherence spectroscopy in the condensed phase: Insights into molecular structure, environment, and interactions," Acc. Chem. Res. 50, 2746-2755 (2017).

${ }^{16}$ G. D. Scholes, G. R. Fleming, L. X. Chen, A. Aspuru-Guzik, A. Buchleitner, D. F. Coker, G. S. Engel, R. van Grondelle, A. Ishizaki, D. M. Jonas, J. S. Lundeen, J. K. McCusker, S. Mukamel, J. P. Ogilvie, A. Olaya-Castro, M. A. Ratner, F. C. Spano, K. B. Whaley, and X. Zhu, "Using coherence to enhance function in chemical and biophysical systems," Nature 543, 647656 (2017).

${ }^{17}$ P. Rebentrost, M. Mohseni, I. Kassal, S. Lloyd, and A. AspuruGuzik, "Environment-assisted quantum transport," New Journal of Physics 11, 033003 (2009).

${ }^{18}$ F. Caruso, A. W. Chin, A. Datta, S. F. Huelga, and M. B. Plenio, "Highly efficient energy excitation transfer in light-harvesting complexes: The fundamental role of noise-assisted transport," J. Chem. Phys. 131, 105106 (2009).

${ }^{19}$ C. M. Isborn, A. W. Götz, M. A. Clark, R. C. Walker, and T. J. Martínez, "Electronic absorption spectra from $\mathrm{mm}$ and ab initio qm/mm molecular dynamics: Environmental effects on the absorption spectrum of photoactive yellow protein," J. Chem. Theory Comput. 8, 5092-5106 (2012).

${ }^{20}$ R. Crespo-Otero and M. Barbatti, "Spectrum simulation and decomposition with nuclear ensemble: formal derivation and application to benzene, furan and 2-phenylfuran," Theor. Chem. Acc. 131, 1237 (2012)

${ }^{21}$ X. Ge, I. Timrov, S. Binnie, A. Biancardi, A. Calzolari, and S. Baroni, "Accurate and inexpensive prediction of the color optical properties of anthocyanins in solution," J. Phys. Chem. A 119, 3816-3822 (2015).

${ }^{22}$ A. V. Marenich, C. J. Cramer, and D. G. Truhlar, "Electronic absorption spectra and solvatochromic shifts by the vertical excitation model: Solvated clusters and molecular dynamics sampling," J. Phys. Chem. B 119, 958-967 (2015).

${ }^{23}$ T. J. Zuehlsdorff, P. D. Haynes, M. C. Payne, and N. D. M. Hine, "Predicting solvatochromic shifts and colours of a solvated organic dye: The example of nile red," J. Chem. Phys. 146 124504 (2017).

${ }^{24}$ J. M. Milanese, M. R. Provorse, E. Alameda, and C. M. Isborn, "Convergence of computed aqueous absorption spectra with explicit quantum mechanical solvent," J. Chem. Theory Comput. 13, 2159-2171 (2017)

${ }^{25} \mathrm{~A}$. Warshel and M. Karplus, "Vibrational structure of electronic transitions in conjugated molecules," Chem. Phys. Lett. 17, 7-14 (1972)

${ }^{26}$ L. S. Cederbaum and W. Domcke, "A many-body approach to the vibrational structure in molecular electronic spectra. I. Theory," J. Chem. Phys. 64, 603 (1976).

${ }^{27}$ K.-M. Chen and C.-C. Pei, "Exact evaluation of two-dimensional Franck-Condon integrals under the Duschinksy mixing effect," Chem. Phys. Lett. 165, 523-527 (1990).

${ }^{28} \mathrm{P}$. T. Ruhrhoff, "Recursion relations for multi-dimensional Franck-Condon overlap integrals," Chem. Phys. 186, 335-374 (1994).

${ }^{29}$ M. Dierksen and S. Grimme, "An efficient approach for the calculation of franck-condon integrals of large molecules," J. Chem. Phys. 122, 244101 (2005)

${ }^{30}$ F. Santoro, A. Lami, R. Improta, J. Bloino, and V. Barone, "Effective method for the computation of optical spectra of large molecules at finite temperature including the duschinsky and herzberg-teller effect: The qx band of porphyrin as a case study," J. Chem. Phys. 128, 224311 (2008).

${ }^{31}$ A. Baiardi, J. Bloino, and V. Barone, "General time dependent approach to vibronic spectroscopy including franck-condon, herzberg-teller, and duschinky effects," J. Chem. Theory Comput. 9, 4097-4115 (2013).

${ }^{32}$ F. Santoro and D. Jaquemin, "Going beyond the vertical approximation with time-dependent density functional theory," Wiley Interdiscip. Rev.: Comput. Mol. Sci. 6, 460-486 (2016).

${ }^{33}$ R. Cammi, S. Corni, B. Mennucci, and J. Tomasi, "Electronic excitation energies of molecules in solution: State specific and linear response methods for nonequilibrium continuum solvation models," J. Chem. Phys. 122, 104513 (2005).

${ }^{34}$ B. Mennucci, "Polarizable continuum model," Wiley Interdisciplinary Reviews: Computational Molecular Science 2, 386-404 (2012).

${ }^{35}$ F. Lipparini and B. Mennucci, "Perspective: Polarizable continuum models for quantum-mechanical descriptions," J. Chem. Phys. 144, 160901 (2016).

${ }^{36}$ T. J. Zuehlsdorff and C. M. Isborn, "Combining the ensemble and Franck-Condon approaches for calculating spectral shapes of molecules in solution," J. Chem. Phys. 148, 024110 (2018).

${ }^{37}$ T. J. Zuehlsdorff and C. M. Isborn, "Modeling absorption spectra of molecules in solution," Int. J. Quantum Chem. 119, e25719 (2019)

${ }^{38}$ T. J. Zuehlsdorff, A. Montoya-Castillo, J. A. Napoli, T. E. Markland, and C. M. Isborn, "Optical spectra in the condensed phase: Capturing anharmonic and vibronic features using dynamic and static approaches," J. Chem. Phys 151, 074111 (2019).

${ }^{39}$ S. Mukamel, Principles of Nonlinear Optical Spectroscopy (Oxford University Press, New York, 1995).

${ }^{40}$ M. C. Zwier, J. M. Shorb, and B. P. Krueger, "Hybrid Molecular Dynamics-Quantum Mechanics Simulations of Solute Spectral Properties in the Condensed Phase: Evaluation of Simulation Parameters," J. Comput. Chem. 28, 1572-1581 (2007).

${ }^{41}$ D. Loco, S. Jurinovich, L. Cupellini, M. F. S. J. Menger, and B. Mennucci, "The modeling of the absorption lineshape for embedded molecules through a polarizable QM/MM approach," Photochem. Photobiol. Sci. 17, 552-560 (2018).

${ }^{42}$ D. Loco and L. Cupellini, "Modeling the absorption lineshape of embedded systems from molecular dynamics: A tutorial review," Int. J. Quantum Chem. 119, e25726 (2018).

${ }^{43}$ A. M. Moran, C. Delbecque, and A. M. Kelley, "Solvent Effects on Ground and Excited Electronic State Structures in the PushPull Chromophore Julolidinyl- $n-N, N^{\prime}$-diethylthiobarbituric Acid," J. Phys. Chem. A 105, 10208-10219 (2001).

${ }^{44}$ A. M. Moran and A. M. Kelley, "Solvent effects on ground and excited electronic state structures of $p$-nitroaniline," J. Chem. Phys. 115, 912 (2001).

${ }^{45}$ A. M. Moran, D. S. Egolf, M. Blanchard-Desce, and A. M. Kelley, "Vibronic effects on solvent dependent linear and nonlinear optical properties of push-pull chromophores: Julolidinemalononitrile," J. Chem. Phys. 116, 2542 (2002).

${ }^{46}$ C. M. Isborn, N. Luehr, I. S. Ufimtsev, and T. J. Martínez, "Excited-State Electronic Structure with Configuration Interaction Singles and Tamm-Dancoff Time-Dependent Density Fucntional Theory on Graphical Processing Units," J. Chem. Theory Comput. 7, 1814-1823 (2011).

${ }^{47}$ T. J. Zuehlsdorff, N. D. M. Hine, J. S. Spencer, N. M. Harrison, D. J. Riley, and P. D. Haynes, "Linear-scaling time-dependent density-functional theory in the linear response formalism," J. Chem. Phys 139, 064104 (2013).

${ }^{48}$ T. J. Zuehlsdorff, N. D. M. Hine, M. C. Payne, and P. D. Haynes, "Linear-scaling time-dependent density-functional theory beyond the Tamm-Dancoff approximation: Obtaining efficiency and accuracy with in situ optimised local orbitals," J. Chem. Phys 143, 204107 (2015).

${ }^{49}$ M. Retegan, F. Neese, and D. A. Pantazis, "Convergence of $\mathrm{qm} / \mathrm{mm}$ and cluster models for the spectroscopic properties of the oxygen-evolving complex in photosystem ii," J. Chem. Theory Comput. 9, 3832-3842 (2013).

${ }^{50} \mathrm{O}$. Valsson, P. Campomanes, I. Tavernelli, U. Rothlisberger, and C. Filippi, "Rhodopsin absorption from first principles: Bypassing common pitfalls," J. Chem. Theory Comput. 9, 2441-2454 
(2013).

${ }^{51}$ M. R. Provorse, T. Peev, C. Xiong, and C. M. Isborn, "Convergence of excitation energies in mixed quantum and classical solvent: Comparison of continuum and point charge models," J. Phys. Chem. B 120, 12148-12159 (2016), pMID: 27797196.

${ }^{52}$ T. J. Zuehlsdorff, P. D. Haynes, F. Hanke, M. C. Payne, and N. D. M. Hine, "Solvent effects on electronic excitations of an organic chromophore," J. Chem. Theory Comput. 12, 1853-1861 (2016).

${ }^{53}$ E. Condon, "A Theory of Intensity Distribution in Band Systems," Phys. Rev. 28, 1182-1201 (1926).

${ }^{54}$ E. Condon, "Nuclear Motion Associated with Electron Transitions in Diatomic molecles," Phys. Rev. 32, 858-872 (1928).

${ }^{55} \mathrm{~S}$. Mukamel, "Fluorescence and absorption of large anharmonic molecules. Spectroscopy without eigenstates," J. Phys. Chem. 89, 1077 (1985).

${ }^{56}$ J. S. Bader and B. J. Berne, "Quantum and classical relaxation rates from classical simulations," J. Chem. Phys. 100, 8359 (1994).

${ }^{57}$ H. Kim and P. J. Rossky, "Evaluation of Quantum Correlation Functions from Classical Data," J. Phys. Chem. B 106, 8240 (2002).

${ }^{58}$ S. A. Egorov, K. F. Everitt, and J. L. Skinner, "Quantum Dynamics and Vibrational Relaxation," J. Phys. Chem. A 103, 94949499 (1999)

${ }^{59}$ I. R. Craig and D. E. Manolopoulos, "Quantum statistics and classical mechanics: Real time correlation functions from ring polymer molecular dynamics," J. Chem. Phys. 121, 3368 (2004).

${ }^{60}$ R. Ramirez, T. Lopez-Ciudad, P. K. P, and D. Marx, "Quantum corrections to classical time-correlation functions: Hydrogen bonding and anharmonic floppy modes," J. Chem. Phys. 121, 3973 (2004).

${ }^{61}$ S. Valleau, A. Eisfeld, and A. Aspuru-Guzik, "On the alternatives for bath correlators and spectral densities from mixed quantum-classical simulations," J. Chem. Phys. 137, 224103 (2012).

${ }^{62}$ V. Helms, C. Winstead, and P. W. Langhoff, "Low-lying electronic excitations in the green fluorescent protein chromophore," J. Mol. Struct. THEOCHEM 506, 179-189 (2000).

${ }^{63}$ S. B. Nielsen, A. Lapierre, J. U. Andersen, U. V. Pedersen, S. Tomita, and L. H. Andersen, "Absorption Spectra of the Green Fluorescent Protein Chromophore Anion in Vacuo," Phys. Rev. Lett. 87, 228102 (2001)

${ }^{64}$ I. B. Nielsen, S. Boyé-Péronne, M. O. A. El Ghazaly, M. B. Kristensen, S. B. Nielsen, and L. H. Andersen, "Absorption Spectra of Photoactive Yellow Protein Chromophores in Vacuuum," Biophys. J. 89, 2597-2604 (2005)

${ }^{65}$ E. Epifanovsky, I. Polyakov, B. Grigorenko, A. Nemukhin, and A. Krylov, "Quantum Chemical Benchmark Studies of the Electronic Properties of the Green Fluorescent Protein Chromophore: 1. Electronically Excited and Ionized States of the Anionic Chromophore in the Gas Phase," J. Chem. Theory Comput. 5, 18951906 (2009).

${ }^{66}$ K. Bravaya and A. I. Krylov, "On the Photodetachment from the Green Fluorescent protein chromophore," J. Phys. Chem. A 117, 11815-11822 (2013).

${ }^{67}$ F. J. A. Ferrer, M. D. Davari, D. Morozov, G. Groenhof, and F. Santoro, "The lineshape of the electronic spectrum of the green fluorescent protein chromophore, part ii: Solution phase," ChemPhysChem 15, 3246-3257 (2014).

${ }^{68}$ T. J. Zuehlsdorff, J. A. Napoli, J. M. Milanese, T. E. Markland, and C. M. Isborn, "Unraveling electronic absorption spectra using nuclear quantum effects: Photoactive yellow protein and green fluorescent protein chromophores in water," J. Chem. Phys. 149, 024107 (2018)

${ }^{69}$ D. A. Case, T. A. Darden, I. Cheatham, C. L. Simmerling, J. Wang, R. E. Duke, R. Luo, R. C. Walker, W. Zhang, K. M. Merz, B. Roberts, S. Hayik, A. Roitberg, G. Seabra, J. Swails, A. W. Götz, I. Kolossvari, K. F. Wong, F. Paesani, J. Vanicek, R. M. Wolf, J. Liu, X. Wu, T. G. H. C. Q. Brozell, S. R.; Stein- brecher, X. Ye, J. Wang, M.-J. Hsieh, G. Cui, D. R. Roe, D. H. Mathews, M. G. Seetin, R. Salomon-Ferrer, C. Sagui, V. Babin, T. Luchko, S. Gusarov, A. Kovalenko, and P. A. Kollman, "Amber 12," (2012), university of California: San Francisco, CA.

${ }^{70} \mathrm{~J}$. Wang, R. M. Wolf, P. A. Caldwell, J. W. an Kollman, and D. A. Case, "Developing and testing of a general amber force field," J. Comput. Chem. 25, 1157-1174 (2004).

${ }^{71}$ W. L. Jorgensen, J. Chandrasekhar, and J. D. Madura, "Comparison of simple potential functions for simulating liquid water," J. Chem. Phys. 79, 926 (1983).

${ }^{72}$ E. D. Getzoff, K. N. Gutwin, and U. K. Genick, "Anticipatory active-site motions and chromophore distortion prime photoreceptor PYP for light activation," Nat. Struct. Biol. 10, 663-668 (2003).

${ }^{73}$ R. C. Walker, M. F. Crowley, and D. A. Case, "The implementation of a fast and accurate $\mathrm{QM} / \mathrm{MM}$ potential method in Amber," J. Comput. Chem. 29, 1019-1031 (2007).

${ }^{74}$ X. He, T. Zhu, X. Wang, J. Liu, and J. Z. H. Zhang, "Fragment quantum mechanical calculation of proteins and its applications," Accounts of Chemical Research 47, 2748-2757 (2014), pMID: 24851673, https://doi.org/10.1021/ar500077t.

${ }^{75}$ H. J. Kulik, J. Zhang, J. P. Klinman, and T. J. Martinez, "How Large Should the QM Region Be in QM/MM Calculations? The Case of Catechol $O$-Methyltransferase," J. Phys. Chem. B 120, 11381-11394 (2016).

${ }^{76}$ M. Karelina and H. J. Kulik, "Systematic quantum mechanical region determination in $\mathrm{qm} / \mathrm{mm}$ simulation," Journal of Chemical Theory and Computation 13, 563-576 (2017), pMID: 28068092, https://doi.org/10.1021/acs.jctc.6b01049.

${ }^{77} \mathrm{~S}$. Hirata and M. Head-Gordon, "Time-dependent density functional theory within the TammDancoff approximation," Chem. Phys. Lett. 314, 291-299 (1999).

${ }^{78}$ T. Yanai, D. P. Tew, and N. C. Handy, "A new hybrid exchangecorrelation functional using the Coulomb-attenuating method (CAM-B3LYP)," Chem. Phys. Lett. 393, 51-57 (2004).

${ }^{79}$ T. H. Dunning Jr., "Gaussian basis sets for use in correlated molecular calculations. i. the atoms boron through neon and hydrogen," J. Chem. Phys. 90, 1007 (1989).

${ }^{80}$ A. Laio, J. VandeVondele, and U. Rothlisberger, "A Hamiltonian electrostatic coupling scheme for hybrid Car-Parrinello molecular dynamics simulations," J. Chem. Phys. 116, 6941 (2002).

${ }^{81}$ D. Das, K. P. Eurenius, and E. M. Billings, "Optimization of quantum mechanical molecular mechanical partitioning schemes: Gaussian delocalization of molecular mechanical charges and the double link atom method," J. Chem. Phys. 117, 10534 (2002).

${ }^{82}$ K. Senthilkumar, J. I. Mujika, K. E. Ranaghan, F. R. Manby, A. J. Mulholland, and J. N. Harvey, "Analysis of polarization in QM/MM modelling of biologically relevant hydrogen bonds," J. Royal Soc. Interface 5, S207-S216 (2008).

${ }^{83}$ F. R. Beierlein, J. Michel, and J. W. Essex, "A simple QM/MM Approach for Capturing Polarization Effects in Protein-Ligand Binding Free Energy Calculations," J. Phys. Chem. B 115, 49114926 (2011).

${ }^{84}$ B. Borucki, H. Otto, T. E. Meyer, M. A. Cusanovich, and M. P. Heyn, "Sensitive Circular Dichroism Marker for the Chromophore environment of Photoactive Yellow Protein: Assignment of the 307 and $318 \mathrm{~nm}$ Bands to the $n \rightarrow \pi^{*}$ Transition of the Carbonyl," J. Phys. Chem. B 109, 629-633 (2005).

${ }^{85} \mathrm{~L}$. Shi and J. L. Skinner, "Proton Disorder in Ice Ih and Inhomogeneous Broadening in Two-Dimensional Infrared Spectroscopy," J. Phys. Chem. A 117, 15536-15544 (2013).

${ }^{86}$ A. M. Rosnik and C. Curutchet, "Theoretical Characterization of the Spectral Density of the Water-Soluble Chlorophyll-Binding Protein from Combined Quantum Mechanics/Molecular Mechanics Molecular Dynamics Simulations," J. Chem. Theory Comput. 11, 5826-5837 (2015).

${ }^{87}$ C. W. Kim, J. W. Park, and Y. M. Rhee, "Effect of Chromophore Potential Model on the Description of Exciton-Phonon Interactions," J. Phys. Chem. Lett. 6, 2875-2880 (2015).

${ }^{88}$ O. Andreussi, I. G. Prandi, M. Canpetella, G. Prampolini, and 
B. Mennucci, "Classical Force Fields Tailored for QM applications: Is it Really a Feasible Strategy?" J. Chem. Theory Comput. 13, 4636-4648 (2017).
${ }^{89}$ S. Yamaguchi, H. Kamikubo, K. Kurihara, R. Kuroki, N. Niimura, N. Shimizu, Y. Yamazaki, and M. Kataoka, "Low-barrier hydrogen bond in photoactive yellow protein," Proc. Natl. Acad. Sci. U.S.A. 106, 440-444 (2009). 